\title{
ON CLOSE-TO-CONVEX FUNCTIONS OF COMPLEX ORDER
}

\author{
H.S. AL-AMIRI and THOTAGE S. FERNANDO \\ Department of Mathematics and Statistics \\ Bowling Green State University \\ Bowling Green, OH 43403, USA \\ (Received November 15, 1988)
}

ABSTRACT. The class $\mathrm{s}^{*}(\mathrm{~b})$ of starlike functions of complex order $\mathrm{b}$ was introduced and studied by M.K. Aouf and M.A. Nasr. The authors using the Ruscheweyh derivatives introduce the class $\mathrm{K}(\mathrm{b})$ of functions close-to-convex of complex order $\mathrm{b}, \mathrm{b} \neq 0$ and its generalization, the classes $k_{n}(b)$ where $n$ is a nonnegative integer. Here $s^{*}(b)$ $c K(b)=K_{0}(b)$. Sharp coefficient bounds are determined for $K_{n}(b)$ as well as several sufficient conditions for functions to belong to $K_{n}(b)$. The authors also obtain some distortion and covering theorems for $K_{n}(b)$ and determine the radius of the largest disk in which every $f \in K_{n}(b)$ belongs to $K_{n}(1)$. All results are sharp.

KEY WORDS AND PHRASES. Starlike functions, close-to-convex functions of complex order, Ruscheweyh derivatives, Hadamard product.

1980 AMS SUBJECT CLASSIFICATION CODE. Primary $30 \mathrm{C} 45$.

1. INTRODUCTION.

Let $A$ denote the class of functions $f(z)$ analytic in the unit disk $E=\{z:|z|<1\}$ having the power series

$$
f(z)=z+\sum_{m=2}^{\infty} a m^{m}, z \varepsilon E .
$$

Aouf and Nasr [1] introduced the class $s^{*}(b)$ of starlike functions of order b, where $b$ is a nonzero complex number, as follows:

$$
S^{*}(\mathrm{~b})=\left\{\mathrm{f}: \mathrm{f} \in \mathrm{A} \text { and } \operatorname{Re}\left[1+\frac{1}{\mathrm{~b}}\left(\frac{z f^{\prime}(z)}{\mathrm{f}(\mathrm{z})}-1\right)\right]>0, z \varepsilon E\right\}
$$

We define the class $\mathrm{K}(\mathrm{b})$ of close-to-convex functions of complex order $\mathrm{b}$ as follows: $f \in K(b)$ if and only if $f \in A$ and

$$
\operatorname{Re}\left\{1+\frac{1}{b}\left(\frac{z f^{\prime}(z)}{g(z)}-1\right)\right\}>0, z \in E
$$

for some starlike function $g$. 
The classes $R_{n}, n \in N_{0}$ and where $N_{0}$ is the set of nonnegative integers, were introduced by Singh and Singh [2], $f \in R_{n}$ if and only if $f \varepsilon A$ and

$$
\operatorname{Re} \frac{z\left(D^{n} f(z)\right)^{\prime}}{D^{n} f(z)}>0, z \in E
$$

where

$$
D^{n} f(z)=f(z) * \frac{z}{(1-z)^{n+1}}
$$

and (*) stands for the Hadamard product of power series, 1.e., if

$$
f(z)=\sum_{0}^{\infty} a_{n} z^{n}, g(z)=\sum_{0}^{\infty} b_{n} z^{n} \text { then } f(z) * g(z)=\sum_{0}^{\infty} a_{n} b_{n} z^{n} .
$$

The operator $D^{n}$ is referred to in A1-Amiri [3] as the Ruscheweyh derivative of order $n$. Note that $R_{0}$ is the familiar class of starlike functions, $S^{*}$. More, it is known [2] that $R_{n+1} \subset R_{n}, n \in N_{0}$, and consequently $R_{n}$ consists of functions starlike in E.

Let $K_{n}(b), n \in N_{0}$, $b$ is a nonzero complex number, denote the class of functions f $\varepsilon$ A satisfying

$$
\operatorname{Re}\left\{1+\frac{1}{b}\left[\frac{z\left(D^{n} f(z)\right)^{\prime}}{D^{n} g(z)}-1\right]\right\}>0, z \varepsilon E,
$$

for some $g \in R_{n}$. Here $K_{0}(b)=K(b)$.

Many authors have studied various classes of univalent and multivalent functions using the Ruscheweyh derivatives $D^{n}, n \in N_{0}$. In particular one can look at the work of Ruscheweyh [4].

Section 2 determines coefficient estimates of functions in $\mathrm{K}_{\mathrm{n}}(\mathrm{b}), \mathrm{n} \in \mathrm{N}_{0}$. In section 3, we obtain some distortion and covering theorems for $k_{n}(b)$ and several sufficient conditions for functions to be in $K_{n}(b)$. The radius of close-to-convexity for the class of close-to-convex of complex order $b$ is also determined in section 3 .

\section{COEFFICIENT ESTIMATES.}

In this section, sharp estimates for the coefficients of functions in $K_{n}(b)$ are determined in Theorem 2.1. First, we need the following lemmas.

LEMMA 2.1. For $\mathrm{n} \in \mathrm{N}_{0}$, let

$$
\left(D^{n} f(z)\right)^{\prime}=\frac{1+(2 b-1) z}{(1-z)^{3}} \text {. }
$$

Then $f \in K_{n}(b)$.

PROOF. Let $g \in A$ be defined so that

$$
D^{n} g(z)=\frac{z}{(1-z)^{2}} \text {. }
$$


The definition of $R_{n}$ implies $g \in R_{n}$. A brief computation gives

$$
1+\frac{1}{b}\left[\frac{z\left(D^{n} f(z)\right)^{\prime}}{D^{n} g(z)}-1\right]=\frac{1+z}{1-z}, z \varepsilon E .
$$

This proves that $f \in K_{n}(b)$.

REMARK 2.1. The function $f$ as defined in (2.1) has the power series representation in $\mathrm{E}$

$$
f(z)=z+\sum_{m=2}^{\infty} \frac{n !(M-1) !}{(n+m-1) !}[(m-1) b+1] z^{m}
$$

LEMMA 2.2. Let $g(z)=z+\sum_{m=2}^{\infty} c_{m} z^{m} \in R_{n}$ where $n \in N_{0}$.

Then $\left|c_{m}\right|<\frac{n ! m !}{(n+m-1) !}$.

PROOF. A brief computation gives

$$
D^{n} g(z)=z+\sum_{m=2}^{\infty} \frac{(n+m-1) !}{n !(m-1) !} c_{m} z^{m} \text {. }
$$

Since $g \in R_{n}, D^{n} g(z) \varepsilon S^{*}$. Thus, using the well known coefficient estimates for starlike functions one gets

$$
\frac{(n+m-1) !}{n !(m-1) !}\left|c_{m}\right|<m, m>2
$$

and the proof is complete.

LEMMA 2.3. Let $f(z)=z+\sum_{m=2}^{\infty} a_{m} z^{m}$. If $f \in K_{n}(b), n \varepsilon N_{0}$, then

$$
\begin{aligned}
& \left|m a_{m}-c_{m}\right|^{2}<4\left[\frac{(m-1) !}{(n+m-1) !}\right]^{2}|b| \\
& \text { - }\left\{n !^{2}|b|+\sum_{k=2}^{m-1}\left[\frac{(n+k-1) !}{(k-1) !}\right]^{2}\left[\left|k a_{k}-c_{k}\right|\left|c_{k}\right|+|b|\left|c_{k}\right|^{2}\right]\right\}
\end{aligned}
$$

PROOF. Let $f(z)=z+\sum_{m=2}^{\infty} a_{m} z^{m}$ be in $K_{n}(b)$. Then (1.5) imp1ies

$$
1+\frac{1}{b}\left[\frac{z\left(D^{n} f(z)\right)^{\prime}}{D^{n} g(z)}-1\right]=\frac{1+w(z)}{1-w(z)}, z \varepsilon E,
$$

for some $g \in R_{n}$ and where $w \in A$ such that $w(0)=0, w(z) \neq 1$ and $|w(z)|<1$ for 
$z \varepsilon$ E. Let $g(z)=z+\sum_{m=2}^{\infty} c_{m} z^{m}$. Then (2.5) and the Definition 1.4 imply

$$
\begin{aligned}
w(z)\{n ! 2 b z & \left.+\sum_{k=2}^{\infty} \frac{(n+k-1) !}{(k-1) !}\left[k a_{k}+(2 b-1) c_{k}\right] z^{k}\right\} \\
& =\sum_{k=2}^{\infty} \frac{(n+k-1) !}{(k-1) !}\left(k a_{k}-c_{k}\right) z^{k} .
\end{aligned}
$$

Using Clunie's method, that is to examine the bracketed quantity of the left-hand side in (2.6) and keep only those terms that involve $z^{k}$ for $k<m-1$ for some fixed $\mathrm{m}$, moving the other terms to the right side, one obtains

$$
\begin{aligned}
w(z)\{n ! 2 b z & \left.+\sum_{k=2}^{m-1} \frac{(n+k-1) !}{(k-1) !}\left[k a_{k}+(2 b-1) c_{k}\right] z^{k}\right\} \\
& =\sum_{k=2}^{m} \frac{(n+k-1) !}{(k-1) !}\left(k a_{k}-c_{k}\right) z^{k}+\sum_{k=m+1}^{\infty} A_{k} z^{k} .
\end{aligned}
$$

Let

$$
\begin{aligned}
\varphi(z) & =w(z)\left\{n ! 2 b z+\sum_{k=2}^{m-1} \frac{(n+k-1) !}{(k-1) !}\left[k a_{k}+(2 b-1) c_{k}\right] z^{k}\right\} \\
& =\sum_{k=2}^{m} \frac{(n+k-1) !}{(k-1) !}\left(k a_{k}-c_{k}\right) z^{k}+\sum_{k=m+1}^{\infty} A_{k} z^{k} .
\end{aligned}
$$
Let $z=r e^{1 \theta}, 0<r<1$. Computing $\frac{1}{2 \pi} \int_{0}^{2 \pi} \varphi(z) \overline{\varphi(z)}$ dz for both expressions of $\varphi(z)$
in (2.7) and using $|w(z)|<1$ we get

$$
\begin{aligned}
& \sum_{k=2}^{m}\left[\frac{(n+k-1) !}{(k-1) !}\right]^{2}\left|k a_{k}-c_{k}\right|^{2} r^{2 k} \\
& <n !^{2} 4|b|^{2} r^{2}+\sum_{k=2}^{m-1}\left[\frac{(n+k-1) !}{(k-1) !}\right]^{2}\left|k a_{k}+(2 b-1) c_{k}\right|^{2} r^{2 k} .
\end{aligned}
$$

Upon letting $\mathrm{r}+1^{-}$and after some easy computations we obtain

$$
\begin{gathered}
\left|m a_{m}-c_{m}\right|^{2}<\left[\frac{(m-1) !}{(n+m-1) !}\right]^{2} 4|b|\left\{n !^{2}|b|+\sum_{k=2}^{m-1}\left[\frac{(n+k-1) !}{(k-1) !}\right]^{2}\right. \\
\left.\cdot\left[\left|k a_{k}-c_{k}\right|\left|c_{k}\right|+|b|\left|c_{k}\right|^{2}\right]\right\} .
\end{gathered}
$$

In particular, when $m=2$ we have

$$
\left|2 a_{2}-c_{2}\right|<\frac{1}{n+1} 2|b| .
$$


The proof of the lemma is complete.

THEOREM 2.1. Let $f(z)=z+\sum_{m=2}^{\infty} a_{m} z^{m}$. If $f \in K_{n}(b)$ where $n \varepsilon N_{0}$,

then

$$
\left|a_{m}\right|<\frac{n !(m-1) !}{(n+m-1) !}[(m-1)|b|+1] \text {. }
$$

This result is sharp. An extremal function is given by (2.3).

PROOF. Let $f(z)=z+\sum_{m=2}^{\infty} a_{m} z^{m}$ be in $K_{n}(b)$. Let the associate function of $f$, $g(z)=z+\sum_{m=2}^{\infty} c_{m} z^{m}$. We claim that for $m>2$ and $n \varepsilon N_{0}$,

$$
\left|m a_{m}-c_{m}\right|<\frac{n !(m-1) !}{(n+m-1)} 2|b|\left[1+\sum_{k=2}^{m-1} \frac{(n+k-1) !}{n !(k-1) !}\left|c_{k}\right|\right] \text {. }
$$

We use the second principle of finite induction on $m$ to prove (2.9).

For $m=2,\left|2 a_{2}-c_{2}\right|<\frac{n !}{(n+1) !} \cdot 2|b|=\frac{2(b)}{(n+1)}$ is true as shown in (2.8). Now assume (2.9) is true for all $\mathrm{m}<\mathrm{p}$. Taking $\mathrm{m}=\mathrm{p}+1$ in (2.4), we get

$$
\begin{aligned}
\left|(p+1) a_{p+1}-c_{p+1}\right|^{2} & <4\left[\frac{p !}{(n+p) !}\right]^{2}|b| \\
& \cdot\left\{n !^{2}|b|+\left[\sum_{k=2}^{p} \frac{(n+k-1) !}{(k-1) !}\right]^{2}\left|k_{k}-c_{k}\right|\left|c_{k}\right|+|b|\left|c_{k}\right|^{2}\right\} \\
& =4\left[\frac{p !}{(n+p) !}\right]^{2}|b|\left\{n !^{2}|b|+\sum_{k=2}^{p}\left[\frac{(n+k-1) !}{(k-1) !}\right]^{2}\left|k a_{k}-c_{k}\right|\left|c_{k}\right|\right. \\
& \left.+|b| \sum_{k=2}^{p}\left[\frac{(n+k-1) !}{(k-1) !}\right]^{2}\left|c_{k}\right|^{2}\right\} .
\end{aligned}
$$

Now using (2.9) since $k<p$, the above yields

$$
\begin{aligned}
& \left|(p+1) a_{p+1}-c_{p+1}\right|^{2}<4\left[\frac { n ! p ! } { ( n + p ) ! } | b | ^ { 2 } \left\{1+2 \sum_{k=2}^{p} \frac{(n+k-1) !}{n !(k-1) !}\left|c_{k}\right|\right.\right. \\
& \cdot\left[1+\sum_{\ell=2}^{k-1} \frac{(n+\ell-1) !}{n !(\ell-1) !}\left|c_{\ell}\right|\right]+\sum_{k=2}^{p}\left[\frac{(n+k-1) !}{n !(k-1) !}\left|c_{k}\right|^{2}\right\} \\
& =4\left[\frac{n ! p !}{(n+p) !}\right]^{2}|b|^{2}\left\{1+2 \sum_{k=2}^{p} \frac{(n+k-1) !}{n !(k-1) !}\left|c_{k}\right|\right. \\
& +2 \sum_{k=2}^{p} \frac{(n+k-1) !}{n !(k-1) !}\left[\left|c_{k}\right| \sum_{\ell=2}^{k-1} \frac{(n+\ell-1) !}{n !(l-1) !}\left|c_{\ell}\right|\right] \\
& \left.+\sum_{k=2}^{p}\left[\frac{(n+k-1) !}{n !(k-1) !}\right]^{2}\left|c_{k}^{2}\right|\right\}
\end{aligned}
$$


Applying the principle of mathematical induction on $p$, it is easily seen that the sum of the last two terms appearing in the bracketed expression in the right hand side of the above is equal to $\left[\sum_{k=2}^{p} \frac{(n+k-1) !}{n !(k-1) !}\left|c_{k}\right|\right]^{2}$. Consequent $1 y$ it follows that

$$
\left|(p+1) a_{p+1}-c_{p+1}\right|^{2}<4\left[\frac{n ! p !}{(n+p) !}\right]^{2}|b|^{2}\left[1+\sum_{k=2}^{p} \frac{(n+k-1) !}{n !(k-1) !}\left|c_{k}\right|\right]^{2} .
$$

This shows that (2.9) is valid for $m=p+1$. Hence, by the second principle of finite induction, the claim is correct. From Lemma 2.2 and 2.9 it follows that

$$
\left|m a a_{m}-c_{m}\right|<\frac{n ! m !(m-1)}{(n+m-1) !}|b|, m>2 \text {. }
$$

Fina11y from Lemma 2.2 and 2.10 we deduce that

$$
\left|a_{m}\right|<\frac{n !(m-1) !}{(n+m-1) !}[(m-1)|b|+1], m>2
$$

Hence the proof of the Theorem 2.1 is complete.

Putting $n=0$ in Theorem 2.1 we have the following corollary.

COROLLARY 2.1. If $f(z)=z+\sum_{m=2}^{\infty} a_{m} z^{m}$ is a close-to-convex function of complex order $b$, then $\left|a_{m}\right|<(m-1)|b|+1$. This result is sharp.

REMARK 2.2. For $b=1$, Corollary 2.1 is reduced to the well known coefficient bounds for the close-to-convex functions due to Reade [5].

Next we have two theorems that provide sufficient conditions for a function to be in $K_{n}(b)$.

THEOREM 2.2. Let $f \in A$ and $n \in N_{0}$. If any of the following conditions is satisfied in $E$, then $f \in K_{n}(b)$.

$$
\operatorname{Re}\left\{1+\frac{1}{b}\left[\left(D^{n} f(z)\right) \cdot-1\right]\right\}>0,
$$$$
\operatorname{Re}\left\{1+\frac{1}{b}\left[(1-z)\left(D^{n} f(z)\right)^{\prime}-1\right]\right\}>0,
$$$$
\operatorname{Re}\left\{1+\frac{1}{b}\left[\left(1-z^{2}\right)\left(D^{n} f(z)\right) \cdot-1\right]\right\}>0
$$

$$
\operatorname{Re}\left\{1+\frac{1}{b}\left[(1-z)^{2}\left(D^{n} f(z)\right)^{\prime}-1\right]\right\}>0 .
$$


PROOF. The proofs follow by choosing $g$ as below:

$$
g(z)=z,
$$

$$
\begin{aligned}
& g(z)=z+\sum_{m=2}^{\infty} \frac{n !(m-1) !}{(n+m-1) !} z^{m}, \\
& g(z)=z+\sum_{m=2}^{\infty} \frac{n !(2 m-2) !}{(n+2 m-2) !} z^{2 m-1}, \text { and }
\end{aligned}
$$

THEOREM 2.3. Let $f(z)=z+\sum_{m=2}^{\infty} a_{m} z^{m}$. For $n \varepsilon N_{0}$, each of the following conditions is sufficient for $f$ to be in $K_{n}(b)$.

$$
\begin{aligned}
& \sum_{m=2}^{\infty} \frac{(n+m-1) !}{n !(m-1) !}\left|a_{m}\right|<|b| \cdot \\
& \sum_{m=2}^{\infty} \frac{(n+m-1) !}{n !(m-1) !}\left|m a_{m}-\frac{(n+m)(m+1)}{m} a_{m+1}\right|<|b|, \\
& 2(n+1)\left|a_{2}\right|+\sum_{m=2}^{\infty} \frac{(n+m-2) !}{n !(m-2) !} \mid(m-1) a_{m-1}-\frac{(n+m)(n+m-1)(m+1)}{m(m-1)} .
\end{aligned}
$$

$a_{m+1}|<| b \mid$, where $a_{1}=1$,

$$
\begin{aligned}
& \text { (1v) } \quad 2\left|(n+1) a_{2}-a_{1}\right|+\sum_{m=2}^{\infty} \frac{(n+m-2) !}{n !(m-2) !} \mid(m-1) a_{m-1}-\frac{2(n+m-1)}{m-1} a_{m} \\
& +\frac{(n+m)(n+m-1)(m+1)}{m(m-1)} a_{m+1}|<| b \mid \text {, where } a_{1}=1 .
\end{aligned}
$$

PROOF. We prove the sufficiency of part (i) since the proofs of the remaining parts are similar to the proof of (i).

From (i) of Theorem 2.2, $f \in K_{n}(b)$ if $f$ satisfies the condition

$$
\operatorname{Re}\left\{1+\frac{1}{b}\left[\left(D^{n} f(z)\right)^{\prime}-1\right]\right\}>0, z \varepsilon E \text {. }
$$

Condition (2.11) would be satisfied if

$$
\left|\frac{1}{b}\left[\left(D^{n} f(z)\right) \cdot-1\right]-1\right|<2, z \in E
$$

is true. However upon substituting

$$
\left(D^{n} f(z)\right)^{\prime}=1+\sum_{m=2}^{\infty} \frac{m(n+m-1)}{n !(m-1) !} a_{m} z^{m-1}
$$

in (2.12) one needs only show 


$$
\left|\frac{1}{b} \sum_{m=2}^{\infty} \frac{m(n+m-1) !}{n !(m-1) !} a_{m} z^{m-1}-1\right|<2, z \varepsilon E
$$

Assuming (i) of this theorem we have

$$
\left|\frac{1}{b} \sum_{m=2}^{\infty} \frac{m(n+m-1) !}{n !(m-1) !} a_{m} z^{m-1}-1\right|<\frac{1}{|b|} \sum_{m=2}^{\infty} \frac{m(n+m-1) !}{n !(m-1) !}\left|a_{m}\right|+1<2 .
$$

Thus (2.13) is established and the proof of the sufficiency of part (1) is complete.

REMARK 2.3. For $n=0$ and $b=1$, Theorems 2.2 and 2.3 are reduced to theorems of Ozaki [6].

3. DISTORTION THEOREMS.

The objective of this section is to obtain some distortion theorems for the class $K_{n}(b)$. The radius of the largest disk $E(r)=\{z /|z|<r\}, 0<r<1$ such that if $f \varepsilon K_{n}(b)$ then $f \varepsilon K_{n}(1)$ can be determined as a consequence of one of those results. THEOREM 3.1. Let $f \in K_{n}(b), n \in N_{0}$. Then for $|z|=r<1$, and $|2 b-1|<1$

$$
\frac{1-|2 b-1| r}{(1+r)^{3}}<\left|\left(D^{n} f(z)\right)\right|<\frac{1+|2 b-1| r}{(1-r)^{3}} \text {. }
$$

This result is sharp. An extremal function $f$ is given by (2.1).

PROOF. Let $f \in K_{n}(b)$. Then (1.5) implies for some $g \in R_{n}$

$$
\frac{z\left(D^{n} f(z)\right)^{\prime}}{D^{n} g(z)}=\frac{1+(2 b-1) w(z)}{1-w(z)}, z \in E,
$$

where $w \in A$ and $|w(z)|<|z|$ in E. This gives for $|z|=r<1$

$$
\frac{1-|2 b-1| r}{1+r}<\left|\frac{z\left(D^{n} f(z)\right)}{D^{n} g(z)}\right|<\frac{1+|2 b-1| r}{1-r} .
$$

The definition of $R_{n}$ implies $D^{n} g(z)$ is a starlike function. Hence by the we11 known bounds on functions which are starlike in $E$, we get for $|z|=r<1$

$$
\frac{r}{(1+r)^{2}}<\left|D^{n} g(z)\right|<\frac{r}{(1-r)^{2}} \text {. }
$$

Using (3.2) together with (3.3) one can get (3.1) and the proof of the Theorem 3.1 is complete.

Taking (1) $\mathrm{n}=0$, and (1i) $\mathrm{n}=0, \mathrm{~b}=1$ in Theorem 3.1 , one can immediately obtain the following corollaries, respectively.

COROLLARY 3.1. If $f$ is a close-to-convex function of complex order $b$ where $|2 b-1|<1$, then for $|z|=r<1$

$$
\frac{1-|2 b-1| r}{(1+r)^{3}}<\left|f^{\prime}(z)\right|<\frac{1+|2 b-1| r}{(1-r)^{3}} .
$$

COROLLARY 3.2. If $\mathrm{f}$ is a close-to-convex function then for $|\mathrm{z}|=\mathrm{r}<1$, 


$$
\frac{1-r}{(1+r)^{3}}<\left|f^{\prime}(z)\right|<\frac{1+r}{(1-r)^{3}}
$$

For the proof of Theorem 3.2, we need the following well known result [7; p. 84] concerning the class $P$ of functions $p(z)$ which are regular in $E$ such that $p(0)=1$ and $\operatorname{Re} p(z)>0, z \varepsilon E$.

LEMMA 3.1. Let $\mathrm{p} \varepsilon \mathrm{P}$. Then for $|\mathrm{z}|=\mathrm{r}<1$,

$$
\left|p(z)-\frac{1+r^{2}}{1-r^{2}}\right|<\frac{2 r}{1-r^{2}} .
$$

This result is sharp.

THEOREM 3.2. Let $f \varepsilon K_{n}(b), n \varepsilon N_{0}$. Then for some $g \varepsilon R_{n}$ and for $|z|=r<1$,

$$
\left|\frac{z\left(D^{n} f(z)\right)^{\prime}}{D^{n} g(z)}-\frac{1+(2 b-1) r^{2}}{1-r^{2}}\right|<\frac{2|b| r}{1-r^{2}} \text {. }
$$

This result is sharp. An extremal function is given in (2.1).

PROOF. $\quad f \in K_{n}$ (b) implies that for some $g \in R_{n}$

$$
1+\frac{1}{b}\left[\frac{z\left(D^{n} f(z)\right)^{\prime}}{D^{n} g(z)}-1\right]=p(z), \quad z \varepsilon E,
$$

where $p \in P$. Hence (3.5) can be obtained by substituting $p(z)$ in $(3.4)$.

It is interesting to note that the result in Theorem 3.2 does not depend on the value of $n$. Also, it can be used to solve the problem concerning the radi1 of $K_{n}$ (b) in $K_{n}(1)$.

THEOREM 3.3. Let $n \in N_{0}$. If $f \in K_{n}(b)$, then $f \in K_{n}(1)$ for $|z|<r$ where

$$
r^{\prime}=\frac{1}{|b|+\sqrt{|b|^{2}-2 \operatorname{Re} b+1}} \text {. }
$$

This result is also sharp. An extremal function is given in (2.1).

PROOF. Let $f \in K_{n}(b)$. Then according to Theorem 3.2 there is some $g \varepsilon R_{n}$ such that for $|z|=r<1, \frac{z\left(D^{n} f(z)\right)}{D^{n} g(z)}$ 11es in the closed disk with center at $\frac{1+(2 b-1) r^{2}}{1-r^{2}}$ and radius $\frac{2|b| r}{1-r^{2}}$. It can be shown that this disk lies in the right half plane if $\mathbf{r}<\mathbf{r}^{\prime}$. This completes the proof of Theorem 3.3.

REMARK 3.1. Taking $n=0$ in Theorem 3.3, one can see that, $r^{\prime}$ is the sharp radius of close-to-convexity for close-to-convex functions of complex order $b$. 


\section{REFERENCES}

1. AOUF, M.K. and NASR, M.A., Starlike Functions of Complex Order b, J. Natural Sc1. Math. 25(1), (1985), 1-12.

2. SINGH, S. and SINGH, S., Integrals of Certain Univalent Functions, Proc. Amer. Math. Soc. 77(3), (1979), 336-340.

3. AL-AMIRI, H.S., On Ruscheweyh Derivatives, Annales Polanic Math., 38(1), (1980), 88-94.

4. RUSCHEWEYH, S., Convolutions in Geometric Function Theory, Les Presses De L'Universite De Montreal (1982)

5. READE, M.0., On Close-to-Convex Univalent Functions, Michigan Mah. J., $\underline{3}$, (1955), 59-62.

6. OZAKI, S., On The Theory of Multivalent Functions, Sc1. Rep. Tokyo Burnika Paig. A2 (1935), 167-188.

7. GOODMAN, A.W., Univalent Functions 1, Marinar Publishing Company Inc.

8. CLUNIE, J., On Meromorphic Schlicht Functions, J. London Math. Soc. 34 (1952), 215-216. 


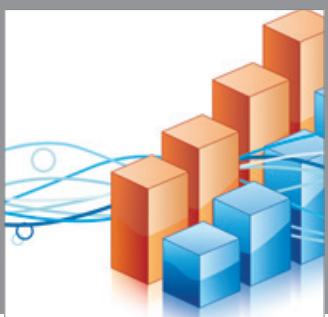

Advances in

Operations Research

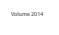

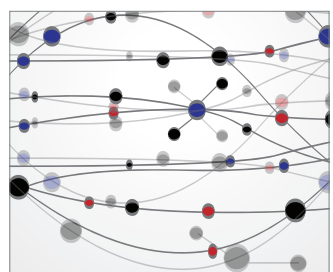

\section{The Scientific} World Journal
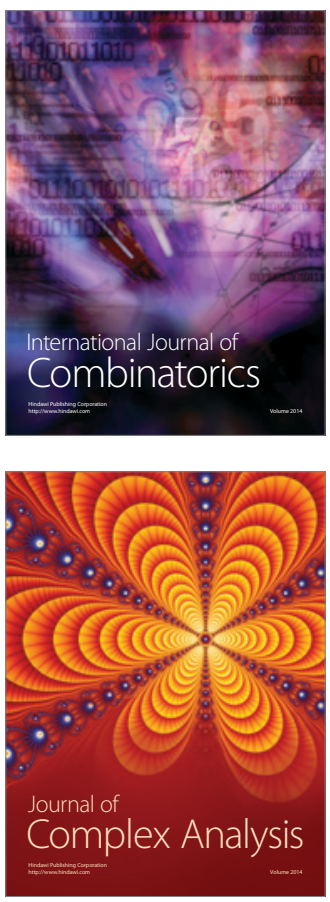

International Journal of

Mathematics and

Mathematical

Sciences
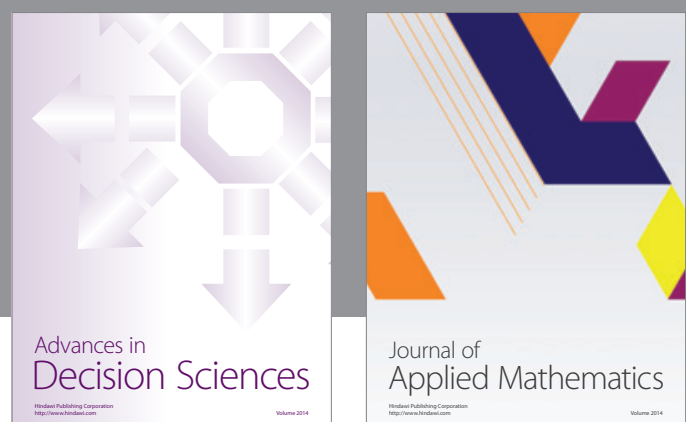

Journal of

Applied Mathematics
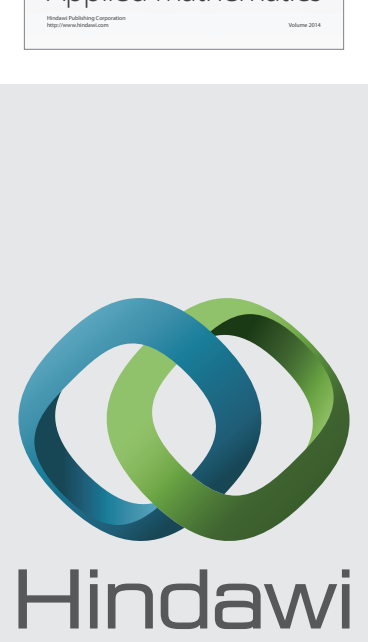

Submit your manuscripts at http://www.hindawi.com
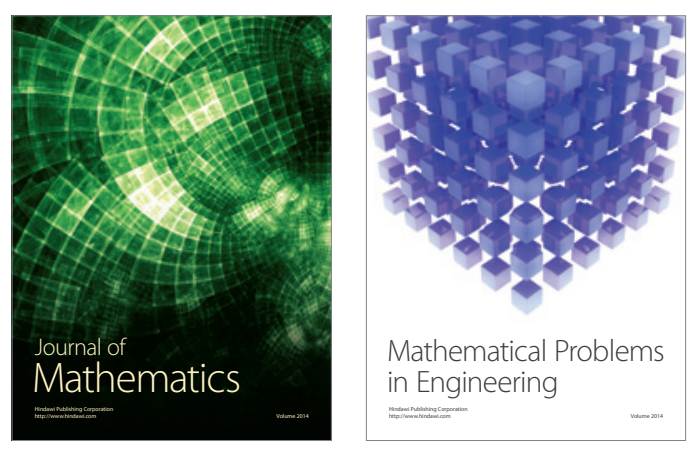

Mathematical Problems in Engineering
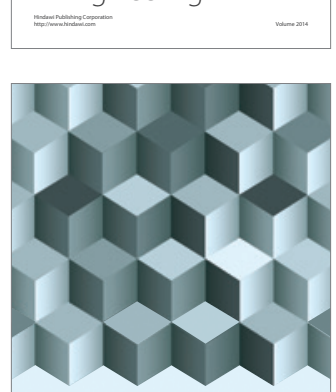

Journal of

Function Spaces
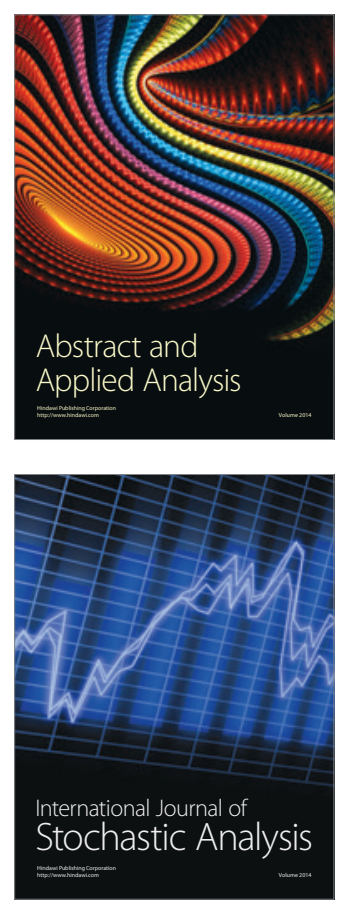

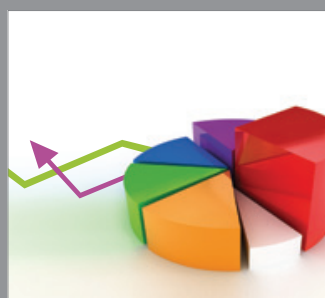

ournal of

Probability and Statistics

Promensencen
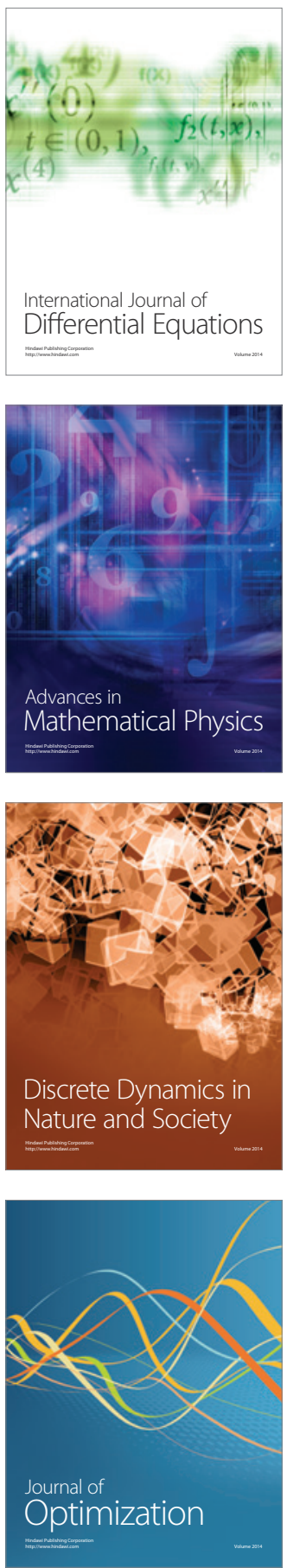